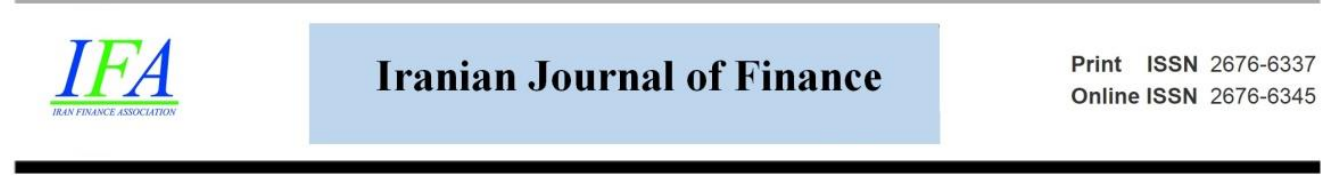

\title{
Portfolio Optimization based on the Risk Minimization by the Weight-Modified CVaR vs. CVaR Method
}

\section{Mohammad Esmaeil Fadaeinejad*}

*Corresponding Author, Associate Prof., Department of Financial Management and Insurance, Shahid Beheshti University, Tehran, Iran. (Email: fadaei@sbu.ac.ir)

\section{Mohammad Taghi Vaziri}

Assistant Prof, Department of Financial Management and Insurance, Shahid Beheshti University, Tehran, Iran. (Email: Amirv7500@gmail.com)

\section{Hossein Asadi}

Associate Prof., Department of Financial Management and Insurance, Shahid Beheshti University, Tehran, Iran. (Email: assadi4@gmail.com)

\section{Mohammad-Javad Faryadras}

Ph.D. Candidate, Department of Financial Management and Insurance, Shahid Beheshti University, Tehran, Iran. (Email: Fariyadras@ gmail.com)

Document Type: Original Article

Received: $2021 / 10 / 20$
2022, Vol. 6, No. 2. 70-94

Published: 2022/02/18

\begin{abstract}
Given the lack of a specific approach to the explanation of values of optimal portfolio weights in the portfolio optimization, the present study aimed to examine large-scale portfolio optimization according to both stock weighting and utilization of SCAD function to minimize the portfolio risk based on the "weight-modified conditional value at risk (CVaR)" and its comparison with
\end{abstract}


the "conditional value at risk $(\mathrm{CVaR}) "$ method in the Tehran Stock Exchange. Therefore, the price information of companies listed in the Tehran Stock Exchange and Over-the-counter (OTC) from 2012 to the end of September 2020 was collected, screened, and analyzed daily, and then the risk and return of the portfolios were examined by forming optimal portfolios. The results indicated that the efficiency limit of the stock portfolio and also the ranks of different companies were different according to the types of the optimization method. Based on the behavior of the TEDPIX, the investors' degrees of risktaking, and the risk management, diversification, and computational complexity of each method, the weight-modified CVaR had a better performance due to better diversification and risk management. Furthermore, the SCAD function added computational complexity to this method.

Keywords: Portfolio Optimization; Conditional value at risk (CVaR); Smoothly-clipped absolute deviation (SCAD) penalty function.

DOI: https://doi.org/ 10.30699/IJF.2021.311328.1281 Publisher: Iran Finance Association

Copyright: author(s)

Type of License: Creative Commons License (CC-BY 4.0)

\section{Introduction}

There are several ways to choose the optimal portfolio. According to the Modern Portfolio Theory, the investors select their portfolios based on the two criteria, namely the expected return and the standard deviation of return. Identifying the factors affecting the selection of the portfolio with a high rate of return and controlled risk has been a topic of interest to researchers. The present research aimed to minimize risk using the conditional value at risk (CVaR), and the CVaR based on the SCAD penalty function that was the weight-modification of the $\mathrm{CVaR}$ method. When it comes to risk minimization, there are several approaches to choosing the optimal portfolio, including Markowitz and Value at Risk (VaR) models. The mean-variance optimization model (MVO) obtains the optimal risk values based on minimizing the variance of total assets in the portfolio based on a certain level of return values (Markowitz, 1952). Markowitz was the founder of a well-known structure called modern portfolio theory. The problem-solving model for choosing an optimal financial portfolio was first proposed by Markowitz (Mohamed, 2005). By defining investment risk quantitatively, Markowitz provided a mathematical approach for investors in asset selection and portfolio management, but as Markowitz and William Sharp acknowledged there are significant constraints on the basic formula in modern portfolio theory. 
Suppose that when the number of shares in the portfolio is large, it will be very difficult or impossible to use the Markowitz method. An important optimization constraint in Markowitz's work is that the sum of the portfolio weights is maximally one that is also called the budget constraint. However, these weights may be extreme and the risk of the portfolio may be high. To eliminate this shortcoming, the present study used the modified $\mathrm{CVaR}$ to prevent extreme weights. The model of optimal portfolio determination within the framework of value at risk is the most important in the sub-branch of PostModern Portfolio Theory (Estrada, 2007). A constraint of the risk-value method is that its approach differs from what we see in real-world portfolio investment assuming the normal risk distribution. In this regard, the method of measuring the conditional value at risk for portfolio optimization has been considered more so that the model seeks to minimize the conditional values at risk in most portfolio optimization functions. Experimental results of various studies in this field also indicate that the conditional value at risk approach offers better performance than traditional approaches such as the Markowitz approach (Karmakar, 2017). As mentioned earlier, the main large-scale optimization problem is the lack of high control over the values of weights, resulting in extreme weights. To control these weights and avoid calculating the extreme weights in portfolio optimization, first, Jagannathan and Ma (2003) sought to control this issue by modifying the proposed approach by Green and Hollifield (1992) and adding a constraint on the extreme values of stock weights in the portfolio. In this way, a SCAD penalty function was added to the portfolio constraint, for instance, studies by Fan \& Li (2001) and Brodie et al. (2009) who tried to reduce this problem by considering the penalty function and adding it to the budget constraint of portfolio optimization for certain extreme weights. To improve this issue in the existing literature, Rockafellar et al. (2014) proposed the generalized least squares regression in the conditional value at risk minimization approach in portfolio optimization problems to reduce problems in extreme weights. Recent studies, such as Xu et al. (2015) also indicated that using the Quantile Regression approach to optimize the Markowitz mean-variance portfolio and the conditional value at risk, unlike the least-squares approach, could take into account different quantiles, operate beyond the mean regression, and provide better results.

Innovations and initiatives of this research include a different mathematical approach in which the explicit solution of the objective function and the conversion of a non-convex problem into a disciplined problem are done. Diversification diagrams also provide a managerial perspective for investors, fund managers, or investment managers that are not considered by 
the researcher in similar research. Comparing the conditional value at risk method and its modified method in the Iranian capital market provides an examination of the ability of each method according to the characteristics of the Iranian financial market and also helps to complete the investment decision-making view. We examined the portfolio optimization with the above two methods among the stocks of the Tehran Stock Exchange and OTC from 2012 to the end of September 2020.

\section{Literature Review}

This section starts with the theoretical bases and then reviews the research literature. Theoretical bases refer to the optimization, Markowitz's method as the creator of the scientific expression of optimization, the introduction of standard deviation as a measure of risk assessment, and limitations of this method, which led to the introduction of value at risk method, shortcomings of the value at risk method, and introduction of conditional value at risk method, and the weight-modified conditional value at risk method, and then examines the research background inside and outside Iran.

The selection of an optimal set of assets is of great importance in economic issues, and the growth and development of this domain. Issues such as the way of risk measurement, the relationship between risk and return, the method of determining the number of shares in the portfolio, and the allocation of assets to companies are important issues for investors. According to modern financial theories, the factors which must be considered to select a suitable portfolio are very important. Rational investment decisions require simultaneous attention to many criteria and different factors (Kocadağlı \& Keskin, 2015). Difficulty in choosing a portfolio is an important topic in financial research. The main purpose of choosing a portfolio is to select the best combination of assets, and the highest expected return and provide an acceptable level of risk (Mokhtar et al., 2014). Choosing the right and optimal portfolio is an issue that all investors, both real and legal, always face. The stock selection includes the creation of a portfolio that maximizes the desirability of the investor. The method of creating such a portfolio has always attracted the attention of financial researchers and analysts. For every investment decision made in any market, there is a very close link between the risk and return of each investment. Therefore, stock portfolio management is the study of all dimensions of stocks, including the composition of shares, the weight of each stock in the portfolio, and the optimal time to change the composition of the portfolio. In addition, stock portfolio management reduces risk and increases returns (Sokhgian \& Valipor, 2010). In this equilibrium of 
risk and return, identifying the efficient frontier of the portfolio of assets allows investors to obtain the highest expected return on their investment based on their utility theory, degree of risk aversion, and risk-taking. Each investor selects a point on the efficient frontier based on their degree of risk-taking and determines the composition of their portfolio to maximize returns and minimize risk (Raei \& Alibeiki, 2010). Portfolio optimization is the selection of the best composition of financial assets in a way that maximizes the return on the investment portfolio and minimizes its risk (Markowitz, 1952).

The problem-solving model for choosing the optimal portfolio was first proposed by Markowitz. The most important drawback of his model is the high number of estimates, leading to the high cost of using his model (Campbell, 2000). Markowitz was the first researcher who scientifically articulated the portfolio and its diversification. He quantitatively proved how the creation of a set of stocks would reduce investment risk. Diversification is the most important part of securities investment management because investors are unsure of the future so they diversify their investments and reduce future risks to reduce the total risk. According to Markowitz's model risk is defined by the dispersion parameter and returns are assumed to be normally distributed, yet the distribution of returns is not always symmetric in practice. According to the above-mentioned cases, variance has been widely criticized as a measure of risk because it treats favorable returns and undesirable negative returns in the same way and considers all dispersions as risk. However, he found that the return on individual assets was more likely to be higher than what was predicted by a normal distribution (Fama \& Roll, 1968). Therefore, the appropriateness of variance as a risk criterion is questionable, and the occurrence of abnormal gains and losses is more than what the normal distribution function predicts. Various criteria have been proposed for risk, many of which are more efficient in measuring risk than variance when the return distribution function is asymmetric. Pedersen et al. (2021) conducted a paper titled "Extended Portfolio Optimization" and pointed out that the average variance method worked much poorly for investors so that investors did not perform the optimization. In the mentioned research, he performed the operational identification of the classical optimization problems and sought to solve them with a simplification and model development approach.

The concept of value at risk was first introduced by Baumol (1963) as a new risk assessment model (Alexander \& Baptistab, 2002), but it has been widely used as a tool for measuring risk since the 1990s. The popularity of this method was due to its simplicity in creating a summary statistical form of potential losses over a time horizon (Mohamed, 2005). The value at risk seeks 
to estimate the reduction of portfolio value from the perspective of market risk. The market risk includes uncertainties about future revenues due to market changes in prices (Kormas, 1998). Even though the value at risk is a very common criterion for calculating risk, it lacks a series of mathematical properties such as addition and convexity and is an inherent criterion of risk only when it is based on a standard deviation from the normal distribution, but the distribution of losses is often not normal in the real world because loss functions tend to show empirical discretization.

The conditional value at risk method was introduced due to the progress of research, and the shortcomings of the above method. Conditional value at risk is the average risk that is greater than the value at risk. In other words, $\alpha \%$ of the mean distribution of the return on the random variable is greater than the value at risk (Rockfellar et al., 2014). Rockafellar \& Uryasev (2002) succeeded in defining the conditional value at risk for general distributions (including discrete distributions) containing a continuous and convex function of portfolio positions. The convexity of this function was an important property of this measure that made the conditional value at risk superior to measures such as value at risk in terms of optimization. More importantly, they found that the uncertain decision-making model decreased to linear programming by describing the behavior of the rate of return on assets in the model by a set of scenarios. Due to the definition and nature of this measure, the conditional value at risk properly observes and estimates the desired risk when faced with skewed and wide-sequence distributions. In addition, it is consistent with the first- and second-order stochastic dominance models for the conditional value at risk (Roman \& Mitra, 2009).

Optimal properties of the conditional value at risk, such as subaggregation, diversification, and coherence capability, significant facilitation of optimization calculations, and control of severe losses have led to the increasing use of this measure in various applications of financial management, for instance, the VaR models introduced by Bogentoft et al. (2002) to manage the assets and liabilities of pension funds, Krokhmal et al. (2002) for risk hedge funds, and Anderson et al. (2002) to optimize credit risk; research also indicates that models based on the conditional value at risk are effective in modeling credit risk and considering the probability of a decline in distribution sequences (Jobst \& Zenios, 2001). This measure has also been used in international portfolio management issues to consider asymmetries of currency rate distribution, and return on assets (Topaloglou et al., 2002). Conditional value at risk is the expected loss of investment provided losses beyond the value at risk (Mohammadi \& Raei, 2008). CVaR is being used in other markets 
such as cryptocurrency to form optimal portfolios, for instance, Pradhan et al. (2021) have used the same method to optimize the market risk of major cryptocurrencies mixed with copula simulation, in which the sample data covers nine cryptocurrencies covering the period of two years and the results using the efficient frontier indicated that if a minimum variance portfolio is constructed using chosen cryptocurrencies, investment in Bitcoin is preferred being the least risky currency on the bottom of the efficient frontier. These results find prime importance for investors and risk managers (Pradhan et al., 2021). The non- assumption of normal risk distribution is the main difference between this method and the value at risk. Giacometti et al. (2021) studied the risk of skewness in large portfolios and used models, which examined the penalty function in quantiles and minimal deviations and proposed quadratic programming to solve optimization problems, and mentioned that real-world analyses on different datasets made it possible to discuss the pros and cons of different optimization methods. The results indicate that the use of the penalty function in linear regression allocations such as Ridge and Elastic Net is more effective in improving the performance of out-of-sample portfolios, especially in large portfolios, compared to those that did not have any penalty function.

Based on the above-mentioned cases and the theoretical and practical success of conditional value at risk on measures such as value at risk, the present research utilizes conditional value at risk as an appropriate risk estimator that has the penalty function in its model function. In addition to measuring its effectiveness, we hope to examine whether, in addition to better measurement of risks in the portfolio of investors active in the Tehran Stock Exchange and OTC, there is evidence of a new and effective measure to financial literature in Iran? Combining this method with the simple conditional value at risk method will provide a better measure of the performance of common methods in the portfolio optimization literature, as tested and reviewed in detail below.

Considering the breadth of experimental literature on portfolio optimization, this section presents the research background based on the objectives. Jagannathan \& Ma (2003) reviewed portfolio optimization by focusing on the extreme values of stock weights in the portfolio. In terms of budget constraints on extreme weights, they found that the constraint in the mean-variance optimization function improved the performance of the meanvariance approach for daily data. Fan \& Li (2001) sought to alleviate problems of extreme weights in large-scale portfolio optimization by considering the penalty function based on the maximum likelihood approach for extreme weights in the portfolio optimization problem. Their studies on this subject led 
to a penalty function called SCAD that acted with better performance than similar optimization approaches. Consistent with the approach proposed by Fan \& Li (2001), Brodie et al. (2001) sought to explain the portfolio optimization using the mean-variance approach considering the penalty function in using linear regression based on penalties. Their results based on the Sharp ratio for comparing different approaches indicated that the use of regression and application of the penalty function had a better performance in the optimization function (Brodie et al., 2001). Instead of explaining the penalty for the optimization problem, $\mathrm{Xu}$ et al. (2015) used the quantile regression approach in the mean-variance models and the conditional value at risk to estimate the optimal weights. Their results for the Chinese stock market on a large scale indicated that the use of the quantum regression approach in optimizing Markowitz's mean-variance portfolio, and the conditional value at risk had better results than the conventional least-squares approach. Ghandehari et al. (2017) introduced conditional value at risk as a criterion for calculating risk in a non-parametric framework and provided an optimal stock portfolio for a certain return and compared the method with the linear programming method. The data in this article consisted of monthly returns of 15 companies selected from the top 50 companies of the Tehran Stock Exchange in the winter of 2013, and they were considered in a period from April 2009 to June 2014. Finally, an optimal portfolio was provided resulting from the use of nonparametric and linear programming methods and their values at risk were compared. It indicated the superiority of the non-parametric method over linear programming. Rostamian et al. (2018) conducted a study titled "Applying the conditional value at risk model in the management of banks' operational risk in the Extreme Value Theory. The results of operational risk calculation indicate that a higher level of confidence increases the conditional value at risk index, and the generalized Pareto distribution (GPD) index provides more reliable results than GEV. Hekmati et al. (2018) examined systematic risk in the financial sector of the Iranian economy (conditional value at risk approach). The results of Quantile regression analysis and previous tests indicated a significant difference between systemic risk and the algebraic sum of the specific risk of each financial sub-sector, including the bank, insurance, and stock exchange. Furthermore, the results of the Friedman test indicated that the insurance industry had the highest share and the banking system had the lowest share in creating systemic risk.

As shown, there are few studies on this field, and they often consider the simple conditional value at risk in the portfolio. Value at risk has also been used frequently in the Iranian financial literature but has shortcomings. This research adds to the literature a more developed measure of what we call the 
weight-modified conditional value at risk, and it solves problems of the conditional value at risk model and tries to improve the model and explicitly solve it, and we then compare it with the value at risk method, which is a wellknown method in the optimization financial literature. As mentioned in other domestic studies, most of the models used to calculate the portfolio risk and optimization approaches have been based on neural networks and genetic algorithms. Many studies can be mentioned in this regard. Therefore, the present study not only sought to fill the existing gap due to the shortcomings of the above-mentioned methods, but also the conditional value at risk was a basis for the research on improved weights, the penalty function was added to the model, and then the investment risk in the portfolio was minimized while explicitly solving the model. Numerous foreign studies also used portfolio constraint approaches, and most of the algorithms were fixed or they used the genetic algorithms and Monte Carlo simulations. A few studies have examined the specific combination of these models with the DE algorithm, and used constraints on the portfolio, especially on weights, for instance, studies by $\mathrm{Xu}$ et al. (2016) as mentioned earlier in the present research.

\section{Research methodology}

The present study was descriptive (non-experimental) due to collecting data realistically without manipulation. Since optimization was examined, it had a survey and exploratory type based on the nature and method. The research used both field and library methods to collect data. To collect the necessary data, it used sources such as documents and library information sources, Internet databases and information portals, Persian and English books, and articles, as well as collecting data from the stock exchange website, and Rahavard Novin software. The subject domain of the research included the stock portfolio optimization based on the modified conditional value at risk method and its simple type. The time-domain of the research was from 2012 to September 2020, and its spatial domain consisted of all companies listed on the Tehran Stock Exchange, and OTC. Due to the wide number of shares in the portfolio, the sample was considered among all Tehran Stock Exchange and OTC companies with the condition that they had more than 215 trading days per year. The lack of share price data for reasons such as blocking or stopping the share should not be more than five months per year. Symbols, which have been closed or stopped for a short period, or their data have not been included in the trading board for any reason, have been approximated and interpolated by the Gaussian second-order interpolation method. 
Therefore, the steps are as follows: 1- Monitoring, summarizing, reviewing, and selecting the sample among the companies listed on the Tehran Stock Exchange and OTC (during the 102 review periods and 231 screened shares); 2- Aggregating the sample stock prices, and dividing by periods to explain and calculate the return vector; 3 - Specifying the optimization problem for the two optimization models introduced in the research; 4-Calculating the covariance matrix by NumPy package in Python; 5- Calculating the optimal stock weight based on the The Gurobi Optimizer in Python; 6- Average return as the input to the model, and its multiplication by weights; 7- Explaining and calculating the penalty function for optimal weights in the modified conditional value at risk model, and then solving it and also the conditional value at risk model without modified weights; 8- Drawing the Pareto front or efficient frontier based on both optimization methods; 9- Drawing the diversification diagram based on each optimization method; 10-Comparing and evaluating the efficiency of the models introduced for the portfolio optimization.

The risk minimization approach is usually performed to optimize the portfolio as follows.

$\left\{\begin{array}{l}\min y^{\prime} \sum y \\ \text { st: } \quad 1^{\prime} w=1\end{array}\right.$

Where the value of the variance-covariance matrix should be considered according to the sum of the weights of the total portfolio assets equal to 1 , and the specified amount of return. In the modern portfolio approach, it is possible to minimize the portfolio risk based on the conditional value at risk method. To this end, we first define the conditional value at risk model as follows.

$$
\operatorname{CvaR}_{\tau}=-E\left[-Y \mid-Y \geq V a R_{\tau}\right]=-\tau^{-1} \int_{0}^{\tau} F^{-1}(t) d t
$$

Where the conditional value at risk is for quantile $\tau$ of the portfolio. The conditional value at risk is operationally equal to $C v a R_{\tau}=\tau^{-1} \min E \rho_{\tau}(Y-$ $\zeta)-\mu$ in which the amount of $\zeta$ of Quantile $\tau$ is equal to $\mathrm{Y}$ and $\rho_{\tau}(\mathrm{u})=$ $\mu(\tau-I(u<0)$. Therefore, the mean values less than the value at risk are equal to the conditional value at risk. Furthermore, $Y=X^{\prime} w$. To apply the penalty function in optimal weights and prevent extreme weights, the following penalty function was obtained according to the study by $\mathrm{Xu}$ et al. (2016). $\sum_{i=1}^{p}$ pen $\left(w_{i}\right) \leq s$ where the amount of $\mathrm{s}$ refers to extreme values in portfolio weighting leading to penalties. Accordingly, this function with the penalty parameter $(\lambda)$ leads to the minimization of the conditional value at risk model 
that is the basis of the difference between this approach and studies conducted in this field in Iran.

$\left\{\begin{array}{l}\min \quad \operatorname{CVaR}_{\tau}(Y)+\lambda \sum_{i=1}^{\rho} \operatorname{pen}\left(w_{i}\right) \leq s \\ s t: 1^{\prime} w=1\end{array}\right.$

Therefore, extreme values in the weights of each portfolio are added to the value of penalty for the conditional value at risk. The sum of these two cases must be minimized in the optimization. The amount of this penalty can be measured according to the SCAD approach introduced by Fan \& Li (2001) as follows:

$\lambda \sum_{i=1}^{p} \operatorname{pen}\left(w_{i}\right)=\sum_{i=1}^{p}\left\langle\lambda\left|w_{i}\right| . I\left(\left|w_{i}\right| \leq \lambda\right\rangle+\left(\left(-w_{i}^{2}+2 \alpha \lambda\left|w_{i}\right|-\lambda^{2}\right) / 2(\alpha-\right.\right.$

1)).I $\left(\lambda<\left|w_{i}\right| \leq \alpha \lambda\right)+\left((\alpha+1) \lambda^{2} / 2\right) . I\left(\alpha \lambda<\left|w_{i}\right|\right)$

Where, I(.) is an indicator function that takes a value of 1 whenever an argument in parentheses is established, otherwise, it is zero. The parameters $\lambda$ and $\alpha$ are explained based on the Bayesian-Schwarz information criterion according to studies by $\mathrm{Li} \& \mathrm{Xu}$ (2013). Therefore, the portfolio maximization can be estimated by considering the minimum conditional risk, and in terms of weight constraint and based on the penalty function. (the same)

$\left\{\begin{array}{l}\min \quad E p_{\tau}\left(x_{1}-\sum_{j=2}^{\rho} X_{j} w_{j}-\xi\right)+\lambda \sum_{i=1}^{p} \operatorname{pen}\left(w_{i}\right) \leq s \\ s t: \quad 1^{\prime} w=1\end{array}\right.$

The constraints in the present study include the constraint-based on optimal weights and conventional models based on the minimization of conditional value at risk. Mathematical models take the values of zero and 1 with their indicator functions and are converted from continuous space to discontinuous and discrete (non-convex) space. The non-convex optimization is the mathematical model of optimization in this method. In the field of nonconvex problems, the Integer linear programming method (ILP) is an appropriate approach to find the answer to the optimization model. To explicitly solve the model, the problem modeling is as follows:

$\operatorname{Min} \quad \operatorname{CVaR}_{\tau}(Y)+\lambda \sum_{i=1}^{s} \operatorname{pen}\left(w_{i}\right) \leq s$ St.

$y_{i} \geq-\sum_{j=1}^{s} \mu_{i j} x_{i}-\eta \quad \forall \mathrm{i}=1, \ldots, n, s$ 


$$
\begin{aligned}
& \sum_{i=1}^{S} x_{i}=1 \rightarrow\left\{\begin{array}{c}
\sum x_{i} \leq 1 \\
\sum x_{i} \geq 1-\varepsilon
\end{array}\right. \\
& \mathrm{i}=1.2, \ldots, \mathrm{s} \\
& 0 \leq x_{i} \leq 1 \\
& \sum_{i=1}^{s} \mu_{i} x_{i} \geq E_{0}
\end{aligned}
$$

Where $\mathrm{i}$ refers to periods, and $\mathrm{j}$ is the number of shares. If we consider the objective function as the delta in this method, then the delta is equal to the sum of the conditional value at risk model with the penalty function. $\mathrm{F}$ is also described in detail mathematically:

$$
\begin{gathered}
\Delta=\eta+\frac{1}{1-\alpha} \sum_{\mathrm{i}=1}^{s} y_{i}+F \\
F=\lambda \sum_{i=1}^{s} \operatorname{pen}\left(w_{i}\right)=\sum_{i=1}^{s}\left\langle\lambda\left|w_{i}\right| . I\left(\left|w_{i}\right| \leq \lambda\right\rangle+\left(\left(-w_{i}^{2}+2 \alpha \lambda\left|w_{i}\right|-\lambda^{2}\right) / 2(\alpha\right.\right. \\
-1)) . \stackrel{I}{I}\left(\lambda<\left|w_{i}\right| \leq \alpha \lambda\right)+\left((\alpha+1) \lambda^{2} / 2\right) . I\left(\alpha \lambda<\left|w_{i}\right|\right)
\end{gathered}
$$

Where, $w_{i}^{2}$ is the formation of a bend or a conical space to get rid of the simplex state and a point of answer and reach a set of answers. It causes portfolio diversification (in mathematics, portfolio diversification is not possible with linear functions). Furthermore, $I\left(\left|w_{i}\right| \leq \lambda\right)$ is an indicator function; and indicator functions are binary or boolean. In other words, they can have values of zero and one for different limits. Therefore, we consider the whole equation of $I\left(\left|w_{i}\right| \leq \lambda\right)$ to simplify and help solve $z_{i(1)}$. We assume the second indicator function of $I\left(\lambda<\left|w_{i}\right| \leq \alpha \lambda\right)$ to be $Z_{i^{(2)}}$, and the third indicator function of $I\left(\alpha \lambda<\left|w_{i}\right|\right)$ to be $z_{i(3)}$ as shown in the following modeling. Now we have to connect the binary variables to $w_{i}$. Therefore, we assume a constraint for $w_{i}$, in which $\mathrm{M}$ is a positive number greater than 1 , to simulate the behavior of the indicator function. We also use $\mathrm{x}$ instead of $w_{i}$ for simplification. $\mathrm{s}$ is the number of assets, and $\mathrm{n}$ is also the number of periods. The SCAD function is defined as follows:

$$
\begin{aligned}
& F=\lambda \sum_{i=1}^{p} \operatorname{pen}\left(w_{i}\right)=\sum_{i=1}^{s}\left(\lambda x_{i^{(1)}}^{\prime}+\left(-x_{i^{(2)}}^{\prime}+2 \alpha \lambda x_{\mathbf{i}^{(2)}}^{\prime \prime}-\lambda^{2} z_{i^{(2)}}\right) /\right. \\
& \left.(2(\alpha-1))+\left((\alpha+1) \lambda^{2} z_{i^{(3)}}\right) / 2\right)
\end{aligned}
$$


The SCAD function in Equation (8) of the present research is the same penalty function because it prevents the creation of portfolios with extreme weights that are not well allocated (Xu et al., 2016). $\lambda$ is a penalty factor. As lambda $(\lambda)$ decreases in a function, which aims to minimize the penalty, portfolio diversification increases. In this method, the problem can be solved by converting it into a disciplined non-convex model with the integer linear programming method in Python. The above constraints can be defined as follows:

$$
\begin{aligned}
& x_{i} \leq \lambda+M\left(1-z_{i^{(1)}}\right), \forall \mathrm{i}=1, \ldots, s \\
& x_{i} \geq \lambda\left(1-z_{i^{(1)}}\right), \forall \mathrm{i}=1, \ldots, s \\
& x_{i}-\lambda \leq(\alpha-1) \lambda+M\left(1-z_{i^{(2)}}\right), \forall \mathrm{i}=1, \ldots, s \\
& x_{i}-\lambda \geq(\alpha-1) \lambda\left(1-z_{i^{(2)}}\right), \forall \mathrm{i}=1, \ldots, s \\
& x_{i} \leq \alpha \lambda+M z_{i^{(3)}}, \forall \mathrm{i}=1, \ldots, s \\
& x_{i}+\varepsilon \geq \alpha \lambda z_{i^{(3)}}, \forall \mathrm{i}=1, \ldots, s \\
& x_{\mathrm{i}^{(1)}}^{\prime} \geq x_{i}-M\left(1-z_{i^{(1)}}\right), \forall \mathrm{i}=1, \ldots, s \\
& x_{\mathrm{i}^{(1)}}^{\prime} \leq z_{i^{(1)}}, \forall \mathrm{i}=1, \ldots, s \\
& x_{\mathrm{i}^{(1)}}^{\prime} \leq x_{i}, \forall \mathrm{i}=1, \ldots, s \\
& x_{\mathrm{i}^{(2)}}^{\prime} \geq x_{i}^{2}-M\left(1-z_{i^{(2)}}\right), \forall \mathrm{i}=1, \ldots, s \\
& x_{\mathrm{i}^{(2)}}^{\prime} \leq z_{i^{(2)}}, \forall \mathrm{i}=1, \ldots, s \\
& x_{\mathrm{i}^{\prime}(2)}^{\prime} \leq x_{i}^{2}, \forall \mathrm{i}=1, \ldots, s \\
& x_{\mathrm{i}^{\prime}(2)}^{\prime \prime} \geq x_{i}-M\left(1-z_{i^{(2)}}\right), \forall \mathrm{i}=1, \ldots, s \\
& x_{\mathrm{i}^{\prime}(2)}^{\prime \prime} \leq z_{i^{(2)}}, \forall \mathrm{i}=1, \ldots, s \\
& x_{\mathrm{i}^{\prime}(2)}^{\prime \prime} \leq x_{i}, \forall \mathrm{i}=1, \ldots, s \\
& x_{i} \in\{0,1\}, y_{i} \in \mathbb{R}, \eta \in \mathbb{R} \\
& x_{\mathrm{i}^{(1)}}^{\prime}, x_{\mathrm{i}(2)}^{\prime}, x_{\mathrm{i}(3)}^{\prime} \in\{0,1\} \\
& z_{i^{(1)}}, z_{i^{(2)}}, z_{i^{(3)}} \in\{0,1\}
\end{aligned}
$$


CVaR model is common in optimization literature, where $y_{i}$ is a variable that calculates the lower limit for the mean return minus $\eta$. It should be noted that since the function is linear and has no curves, the model finds the extreme points and based on which performs the calculations. But in the modified CVaR model, by converting these extreme points in the polyhedral to curves (convex composition), all points that are in line with the target function gradient will be selectable, in which case the optimal solution will not be limited to one, two, three and/or a limited number of shares, but a vector of shares. This is what is done in the mathematical concept of a modified CVaR on weights, somewhat different from the simple CVaR method. Also, when coding in Python in the portfolio optimization domain, the expression $\sum_{i=1}^{S} x_{i}=1$ is not exactly equal to one to avoid solver face problems. This expression, as follows, is divided into two categories, larger and smaller equal to one, which is considered as a functional constraint so that solver will not have trouble satisfying equal constraints and will find the answer with a difference of one epsilon. The last limitation solves the model in Python by increasing different $E_{0}$, and different answers will be obtained in different iterations (test times) for the minimum and higher $E_{0}$.

Min $\quad \eta+\frac{1}{1-\alpha} \sum_{i=1}^{s} y_{i}$

St.

$y_{i} \geq-\sum_{i=1}^{s} \mu_{i} x_{i}-\eta$

$\sum_{i=1}^{S} x_{i}=1 \rightarrow\left\{\begin{array}{c}\sum x_{i} \leq 1 \\ \sum x_{i} \geq 1-\varepsilon\end{array}\right.$

$\mathrm{i}=1.2, \ldots, \mathrm{s}$

$\sum_{i=1}^{s} \mu_{i} x_{i} \geq E_{0}$

$0 \leq x_{i} \leq 1$

\section{Data Analysis and results}

Each of the methods has a computational Excel file, a Pareto front or efficient frontier table, and a portfolio diversification table based on risk-return balance preferences. The Excel file, which contains the information extracted from Python software, has 231 symbols as the input of the model. The numbers 
inside the Excel file cells are weights. The sum of the columns observes the condition that the sum of the weights of the portfolio is equal to 1 . We obtain the weights by adding the constraints in a way that the sum of the weights is equal to 1. Our optimization engine is an American software package called the Gurobi Optimizer, which provides academic access and has libraries on Python software and is used to gain weights and optimize along with Python software codes. A mathematical contribution of this research is to explicitly solve the weight-modified conditional value at risk model, while $\mathrm{Xu}$ et al. (2015) simulated and solved a pathway using the Monte Carlo simulation method for each Lambda in the original model. It is noteworthy that the value at risk and conditional value at risk models are classified as stochastic programming and cannot be explicitly solved, and will be solved either by simulation or, like the present research, by converting these models into disciplined models by linearization or graded models. Another creativity of the research is to draw a portfolio diversification chart for each method, which is not found in any similar work, while this chart can give a managerial insight into what stocks will be selected at each level of risk and return. The table below illustrates the Pareto front or the efficient frontier in the weight-modified conditional value risk method. As shown, this method shows the behavior of the model by providing two diagrams of the Pareto front, and consequently the portfolio diversification diagram.

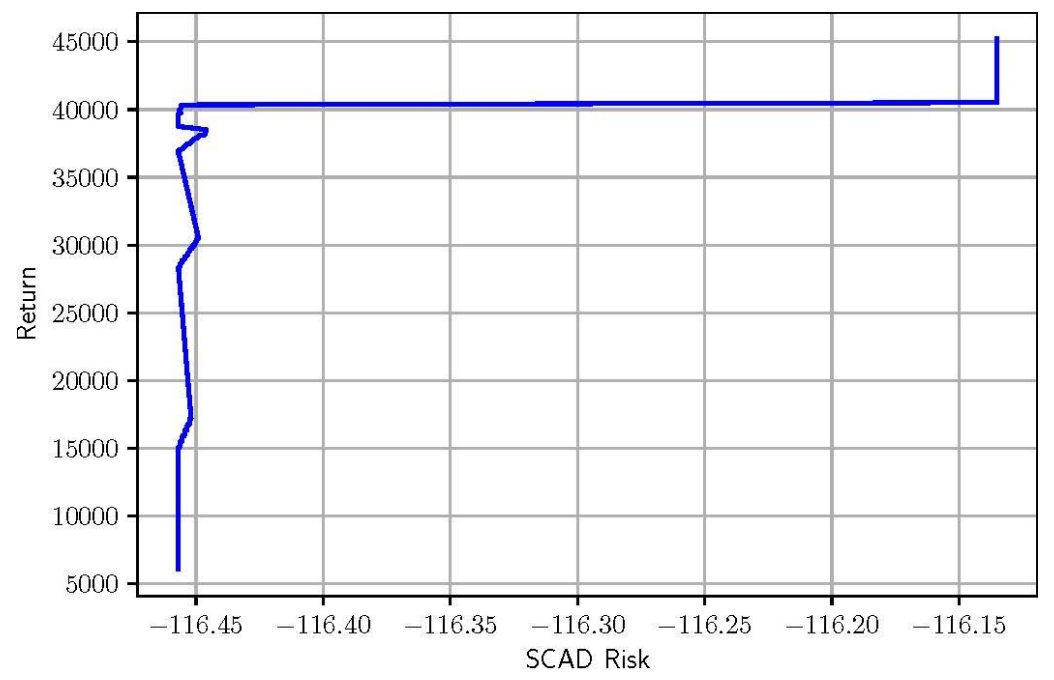

Figure 1. The efficient frontier or Pareto front with weight-modified conditional value at risk method 
Portfolio Optimization based on the Risk Minimization by...

Figure 1 shows the extreme state of the model behavior. In all models of both methods, we see that the price return is drawn from 5 to 45 thousand units, and if it is outside this range, the model becomes non-operational, and the solver encounters an error. The horizontal axis of the diagram is the same as the objective penalty function. In this diagram, it is noteworthy that the SCAD value of the model is robust based on all efficiencies. In other words, the risk is kept at a level, and returns change. The risk ranges from -116.45 to 116.15 in the horizontal axis of the diagram. It can be interpreted that these numbers while being significant, have a significant risk or the value of SCAD Risk is robust for any return and does not change. Diagram 2 shows a magnified part of Diagram 1. If two different returns are available for the investor to access at a certain level of risk, it is logical that a higher level of return will be chosen [41]. Diagram 3, which relates to the situation in which a larger rate of return is considered, this model recommends selecting seven stocks with the lowest level of risk and rate of return. Figure 4 magnifies the diversification in the range of the first efficient front in which the optimal portfolio of the investor varies from 5 to 8 shares.

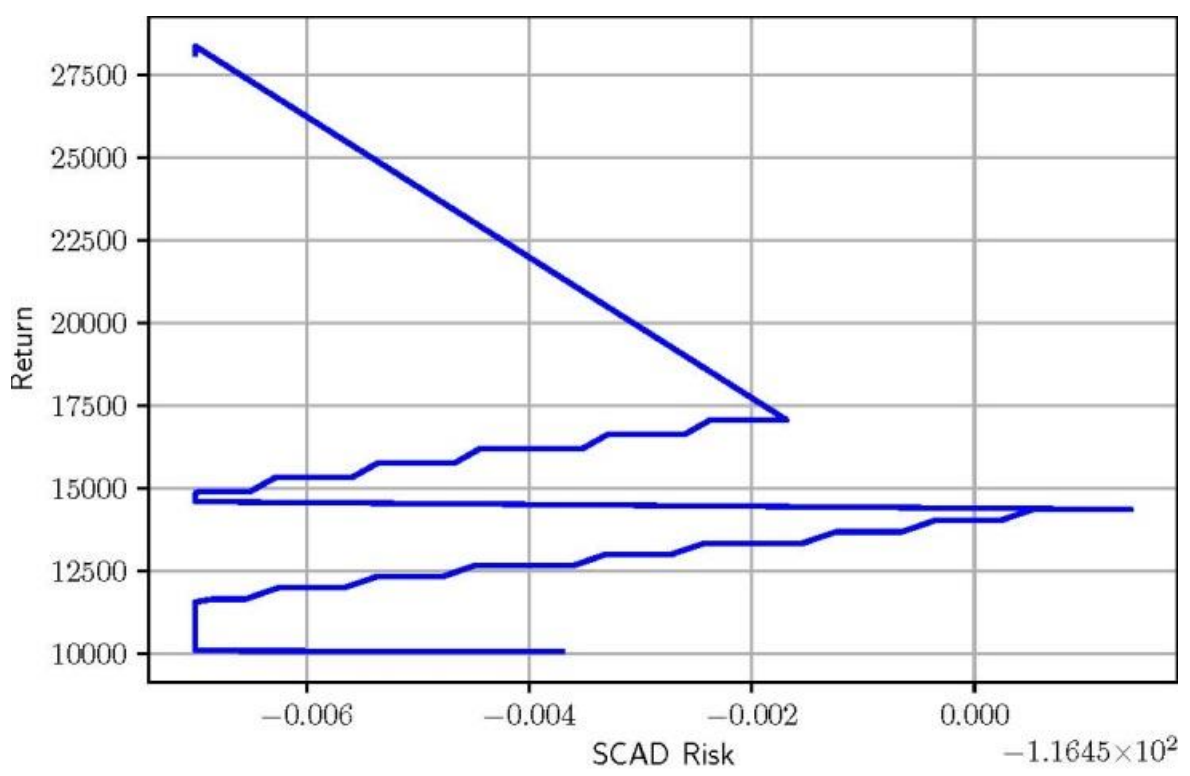

Figure 2. Efficient frontier or magnified Pareto front 


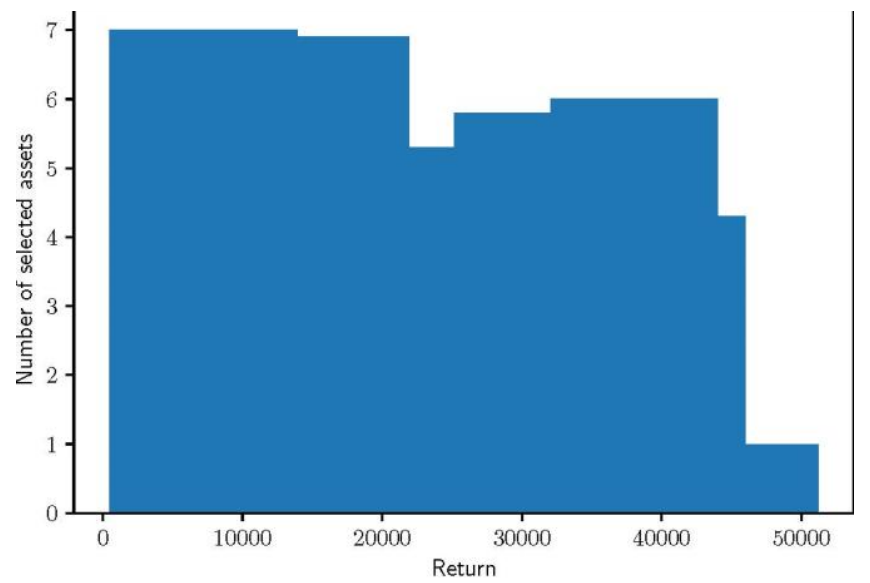

Figure 3. Diversification of the portfolio by the weight-modified conditional value at risk method

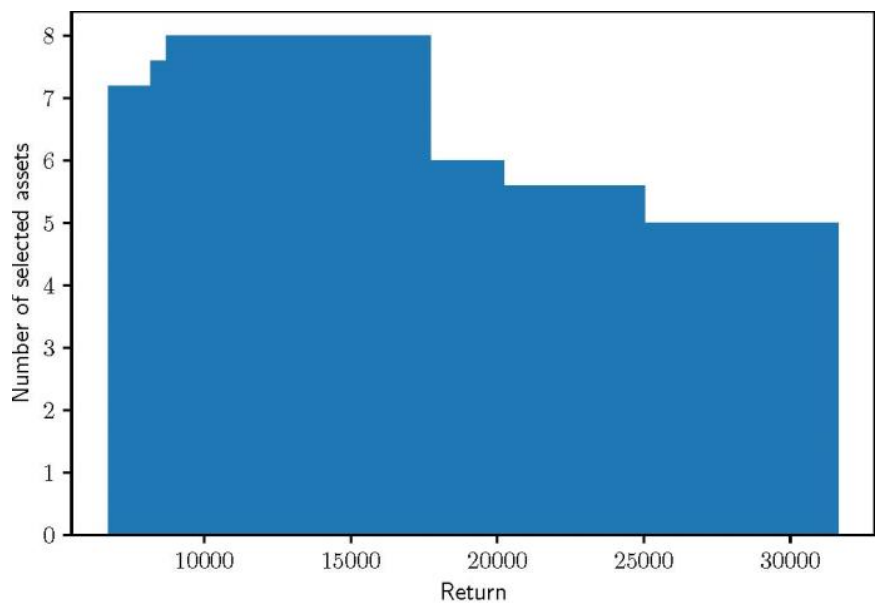

Figure 4. Diversification of the portfolio of the same method in a magnified mode

Figure 5 illustrates the Pareto front or the efficient frontier in the conditional value at risk method. As shown, the Pareto front has a linear behavior. We could assume the same speculation about the Pareto front of the value-at-risk model because its objective function was also linear. In this model of optimization methods, the efficiency has a range of 5 thousand to 45 thousand units. 
Portfolio Optimization based on the Risk Minimization by...

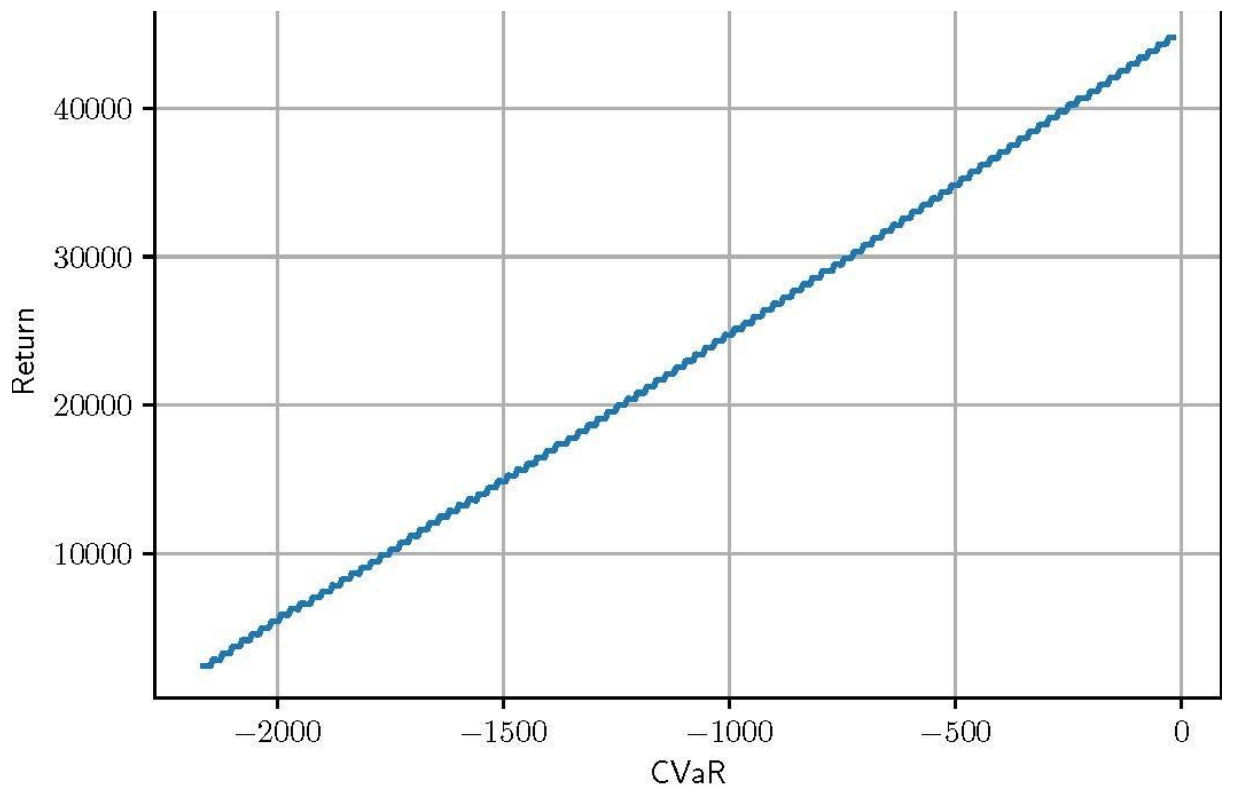

Figure 5: Efficient frontier or Pareto front with conditional value at risk method

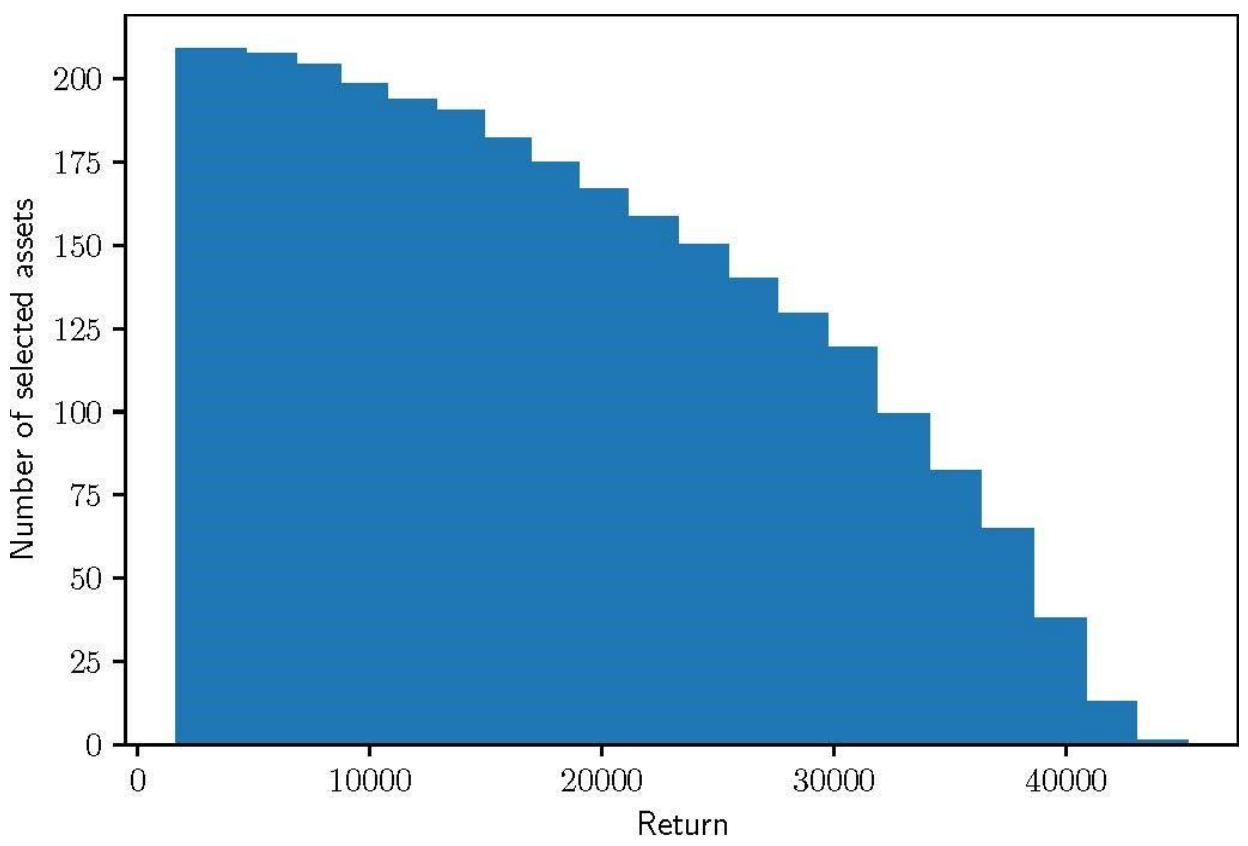

Figure 6: Portfolio diversification by conditional value at risk method 


\section{Discussion and Conclusion}

The more shares are selected in the diversified portfolio, the lower the portfolio risk and the lower the expected return for the investor or fund manager [42]. The efficient frontier between the modified conditional value at risk method and the conditional value at risk method is different in terms of various reasons but both are drawn based on the lowest rate of return, or return floor desired by the investor. This means that solving the optimizer model at different levels of risk can result in the minimum return that the investor can obtain. According to the test of these two methods and considering different levels of risk and return, we see that the diversification in each method was different from the other. In the weight-modified conditional value at risk method, 6 to 7 shares are introduced for a minimum return up to a level of 4000 units, and then in the riskiest case, a share is introduced to achieve 50,000 units of return considering the penalty function. It is pointed out that the second diversification diagram in the above-mentioned method is drawn in a magnified form due to the special mode of its efficient frontier diagram, which is not the usual mode; and it has the risk, and return range more limited to the oscillating part of the efficient frontier. In this case, pulling out a part of the efficient frontier, which has an extreme mode, and the diagram is magnified to a return of 1000 to 3000 units, the risk management and diversification are more logical than the previous case, and 5 to 8 selected shares are introduced while the weights are not extreme.

Among the two methods, the weight-modified conditional value at risk method had a better performance in terms of risk management, portfolio diversification, and non-extreme weights. However, the computational complexity of this method is a problem. Combining the conditional value at risk method with the penalty function results in providing levels of return, which are robust to risk, for investors and these levels of return are more likely to be gained. Table 1 summarizes a comparison of the two methods.

Table 1. Comparison of optimization methods in the present research

\begin{tabular}{|c|c|c|}
\hline Method & $\begin{array}{c}\text { Weight-modified conditional } \\
\text { value risk method }\end{array}$ & $\begin{array}{c}\text { Conditional value risk } \\
\text { method }\end{array}$ \\
\hline Risk management & Above medium & Medium \\
\hline Portfolio diversification & Very strong & Weak \\
\hline Computational complexity & High & Low \\
\hline
\end{tabular}


The weight-modified conditional value at risk method has higher-thanaverage risk management because it improves the conditional value at risk method as it has a high degree of diversification due to the penalty function, and consequently, the model solution has relatively high complexity. This computational complexity is partly related to the SCAD model conversion to an explicit model. As a non-convex model with the linearization method, SCAD is convex and can be solved directly without simulation by converting to disciplined models that consequently have difficult calculations. However, the conditional value at risk method observes and estimates the desired risk well due to the definition and nature of this measure when dealing with skewed and wide-sequence distributions of the conditional value at risk [43]. The results were also consistent and indicated that the risk and asset management of this method alone were good, but it was considered moderate in comparison with other methods because it was a method between the mean-variance of conditional value at risk method and the value at risk method. Portfolio diversification of this method is weak because it considers the extreme optimization problem, while the model function of this method is also linear and does not have the capabilities of more complex models that consider more comprehensive approaches. The low linearity of the model is its difficulty, and the model of this method is easier to be solved than conventional methods. To use these two methods in the Iranian capital market, two scenarios are presented about the behavior of the total index as a thermometer of the Iranian capital market. If the overall volatility index is high, the situation is different from the robust state. In the first scenario, when we see the volatility of the total index, it is recommended that the rational investor (not highly risk-taking/ risk-averse) should accept less risk and buy more symbols, and when the volatility of the total index is low, we can accept higher risk and fewer stocks to achieve higher returns. In the first scenario, it is suggested that if the volatility of the total index is high, the modified conditional value at risk method (the conditional value at risk in the second priority) should be used because the modified method has better risk management and diversification. In the second scenario, if the market is robust, bearish or bullish, we can use mean-variance methods such as mean-variance of conditional value at risk that focuses on maximizing returns (not minimizing risk). The reason for this issue is visible in the Iranian capital market due to the investors' emotional behaviors. Whether the market is completely bullish or completely bearish over a period of one to three months, portfolio diversification will practically lose its operational nature due to the locking of the stock market in the buying and selling queues. The researcher believes that giving a definitive answer to a dynamic problem over time will not have scientific accuracy. 


\section{Suggestions and limitations}

The concept of portfolio optimization and diversification is the basis of financial market expansion and financial decision-making [44f]. In the present study, the penalty function is added to the weight-modified conditional value at risk model to improve the target model and prevent extreme weights. The objective function consists of two functions, the conditional value at risk and the penalty function, and does not have the mean return criterion. This model can be developed creatively. Future researchers are suggested to include the return element in the function $\mathrm{F}$ (Equation (8) in the present research) by observing the scientific requirements of the problem. It is common to use multi-objective methods in solving financial and optimization problems, but since risk and return are not the same things, we can achieve the model development by adding a value function 1 to the optimization problem that matches the values of risk and returns. If future researchers can create a bend in the target function and add it to the model (previously we only had risk minimization in the portfolio optimization function with a weight-modified conditional value at risk method), and the function can provide different contributions for multiple returns averages by the model development, it seems that a significant model contribution will be made to achieve returns in the objective function because this model offers a different portfolio for a certain level of risk.

Conducting research based on dynamic programming is the second research proposal, indicating limitations of the present research, and the mathematical optimization models. The issue of stochastic dynamic portfolio optimization is another topic for future research. It should be noted that the methods used in this study all consider all periods together, but the optimized portfolio can be built in the recursive functions at the end of each period (it can be two weeks, a month, or so on depending on the research), and the cumulative wealth can be calculated for each period, and we can have a portfolio for the next period rearranged by buying, selling, or maintaining the stocks.

If future researchers can design a method for the capital market in Iran to add a series of known uncertainties, and a series of predetermined assumptions to the model, we can see in the statistics that the researcher will receive more realistic answers from the optimized model by limiting the range of

\footnotetext{
${ }^{1}$ The value function of a problem gives the optimization of the value obtained by the objective function in a solution, while it depends only on the parameters of the problem.
} 
fluctuations and adding default information to the time series or series being studied and researched because these types of defaults are not hidden in the model, and the researcher can make a better prediction by adding them to the model (e.g., the amplitude of fluctuation is between -5 and +5). Future researchers can increase the allowable fluctuation range on stock returns to determine if the model exceeds the reported number, and obtain the necessary resiliency model against this change. Such research on the Iranian capital market-focused academic environment is a state-of-the-art model that needs further research. None of these models takes into account the fluctuation range that is examined daily, which is assumed to be in the range of -5 to +5 . These models cannot include these fluctuations as prior information in the input data, and it must be understood that assuming the model predicts for the next day does not exceed the fluctuation amplitude by entering such data into the model. In this regard, an approach is to use the uncertainty sets that are proposed in the field of robust optimization by which the data can be used as an input to the model in a model such as the Markowitz mean-variance model to provide real robustness. Therefore, the robustness of mathematical models without such inputs is mostly pessimistic in building uncertainty and prediction-based models, while the future researcher in the Iranian capital market must provide realistic predictions that are not in the mathematical models. The prediction is a key point in building uncertainty models so that the model conservatism should be reduced (not modeled in a way that it is extremely risk-averse), and the model should be parsimony, meaning that it has the least amounts of variables and parameters to describe the model. The convex uncertainty sets are the best way to do this style of research.

The third domain for future research includes the research that researchers provide new researchers with the new optimization areas by adding default information to the model and using Bayesian mathematics and combining it with Markowitz's method and other optimization methods. Therefore, a part of the model can be developed according to Bayesian mathematics, and the preliminary information can be given as input to the model, and more realistic predictions can be provided.

\section{Declaration of Conflicting Interests}

The authors declared no potential conflicts of interest concerning the research, authorship and, or publication of this article.

\section{Funding}

The authors received no financial support for the research, authorship and, or publication of this article. 


\section{References}

Alexander, G. J., \& Baptista, A. M. (2002). Economic implications of using a meanVaR model for portfolio selection: A comparison with mean-variance analysis. Journal of Economic Dynamics and Control, 26(7-8), 1159-1193.

Brodie, J., Daubechies, I., De Mol, C., Giannone, D., \& Loris, I. (2009). Sparse and stable Markowitz portfolios. Proceedings of the National Academy of Sciences, 106(30), 12267-12272.

Campbell, J. Y. (2000). Asset pricing at the millennium. The Journal of Finance, 55(4), 1515-1567.

Estrada, J. (2007). Mean-semivariance behavior: Downside risk and capital asset pricing. International Review of Economics \& Finance, 16(2), 169-185.

Fama, E. F., \& Roll, R. (1968). Some properties of symmetric stable distributions. Journal of the American Statistical Association, 63(323), 817-836.

Fan, J., \& Li, R. (2001). Variable selection via nonconcave penalized likelihood and its oracle properties. Journal of the American Statistical Association, 96(456), 1348-1360.

Ghandehari, M., Shamshiri, A., Fathi, S. (2017). portfolio optimization based on nonparametric estimation methods. Journal of Production and Operations Management, 8(1), 175-184. (In Persian)

Giacometti, R., Torri, G., \& Paterlini, S. (2021). Tail risks in large portfolio selection: penalized quantile and expectile minimum deviation models. Quantitative Finance, 21(2), 243-261.

Green, R. C., \& Hollifield, B. (1992). When will mean-variance efficient portfolios be well-diversified?. The Journal of Finance, 47(5), 1785-1809.

Hekmati Farid, S., Rezazadeh, A., Malek, A. (2018). The Estimation of Systematic Risk in Iranian Financial Sectors ( $\triangle \mathrm{CoVaR}$ Approach). Economic Modeling, 12(43), 99-122. (In Persian)

Jagannathan, R., \& Ma, T. (2003). Risk reduction in large portfolios: Why imposing the wrong constraints helps. The Journal of Finance, 58(4), 1651-1683.

Jobst, N. J., \& Zenios, S. A. (2001). The tail that wags the dog: Integrating credit risk in asset portfolios. The Journal of Risk Finance.

Karmakar, M. (2017). Dependence structure and portfolio risk in Indian foreign exchange market: A GARCH-EVT-Copula approach. The Quarterly Review of Economics and Finance, 64, 275-291.

Kormas, G. (1998). Daily and intradaily stochastic covariance: value at risk estimates for the foreign exchange market (Doctoral dissertation, Concordia University). 
Li, J., \& Xu, M. (2013). Optimal dynamic portfolio with Mean-CVaR criterion. Risks, 1(3), 119-147.

Markowitz, Harry, M. (1952). Portfolio Selection, Journal of Finance, 7 (1), 77-91.

Markowitz, Harry, M., (1959). Portfolio Selection: Efficient Diversification of Investments, New York: John Wiley \& Sons.

Mokhtar, M., Shuib, A., \& Mohamad, D. (2014). Mathematical programming models for portfolio optimization problem: A review. International Journal of Social, Management, Economics and Business Engineering, 8(2), 443-450.

Mohamed, A. R. (2005). Would students T-GARCH improve VaR estimates? Master Thesis, University of Jyvaskyla, Finland.

Mohammadi, S., Raei, R., \& Faizabad, A. (2008). Forecasting value-at-risk using conditional volatility models: Evidence from Tehran stock exchange. Financial Research Journal, 10(25), 109-124.

Nikoo, S., Shams, S., Seyghali, M. (2020). Modeling of Optimal Stock portfolio Optimization Based on Risk Assessment and Behavioral Financial Approach (Mental Accounting) in Tehran Stock Exchange. -Journal of Financial Management Perspective, 10(31), 75-101. (In Persian)

Kocadağlı, O., \& Keskin, R. (2015). A novel portfolio selection model based on fuzzy goal programming with different importance and priorities. Expert Systems with Applications, 42(20), 6898-6912.

Pedersen, L. H., Babu, A., \& Levine, A. (2021). Enhanced portfolio optimization. Financial Analysts Journal, 77(2), 124-151.

Pradhan, A. K., Mittal, I., \& Tiwari, A. K. (2021). Optimizing the market-risk of major cryptocurrencies using $\mathrm{CVaR}$ measure and copula simulation. Macroeconomics and Finance in Emerging Market Economies, 117.

Raei, R., Alibeiki, H. (2010). Portfolio optimization using particle swarm optimization method. Financial Research Journal, 12(29), (In Persian)

Rahnamay Roodposhti, F., Nikoomaram, H., Toloei, A., Hoseinzadeh Lotfi, F., (2017). Evaluation of portfolio optimization performance using maximum stable Sharp ratio compared to Markowitz optimization. -Journal of Financial Management Perspective, 7(18), 125-145. (In Persian)

Rockafellar, R. T., Royset, J. O., \& Miranda, S. I. (2014). Superquantile regression with applications to buffered reliability, uncertainty quantification, and conditional value-at-risk. European Journal of Operational Research, 234(1), $140-154$.

Roman, D., \& Mitra, G. (2009). Portfolio selection models: A review and new directions. Wilmott Journal: The International Journal of Innovative 
Quantitative Finance Research, 1(2), 69-85.

Rostamian, N., Hoseini Yekani, A., Kashiri Kolaei, F., (2018). Optimization of Major Bank Balance Sheet Items in Iran, National Conference on New Studies in Economics, Management and Accounting in Iran, (In Persian)

Sokhgian, M. \& Valipor, H. (2010), Multi-criteria decision-making method for selecting stocks on the stock exchange using financial variables, Financial Engineering Pub., 1(5), 35-51. (In Persian)

Topaloglou, N., Vladimirou, H., \& Zenios, S. A. (2002). CVaR models with selective hedging for international asset allocation. Journal of Banking \& Finance, 26(7), 1535-1561.

Xu, Q., Niu, X., Jiang, C., \& Huang, X. (2015). The Phillips curve in the US: A nonlinear quantile regression approach. Economic Modelling, 49, 186-197.

Xu, Q., Zhou, Y., Jiang, C., Yu, K., \& Niu, X. (2016). A large CVaR-based portfolio selection model with weight constraints. Economic Modelling, 59, 436-447.

\section{Bibliographic information of this paper for citing:}

Fadaeinejad, Mohammad Esmaeil; Vaziri, Mohammad Taghi; Asadi, Hossein \& Faryadras, Mohammad-Javad (2022). Portfolio Optimization based on the Risk Minimization by the Weight-Modified CVaR vs. CVaR Method. Iranian Journal of Finance, 6(2), 70-94.

Copyright @ 2022, Mohammad Esmaeil Fadaeinejad, Mohammad Taghi Vaziri, Hossein Asadi and Mohammad-Javad Faryadras

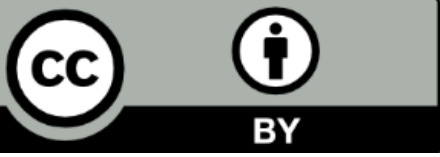

